\title{
Multipotent stem cells with neural crest stem cells characteristics exist in bovine adipose tissue
}

\author{
Kai Zhang a, b, \#, Xiao-zhen Cui ${ }^{\text {b, }}$, Bo-chi Zhang ${ }^{\text {b }}$, Xian-yi Song ${ }^{\mathrm{b}}$, Qiang Liu ${ }^{\text {a, }}$, Shi-yu Yang c, *

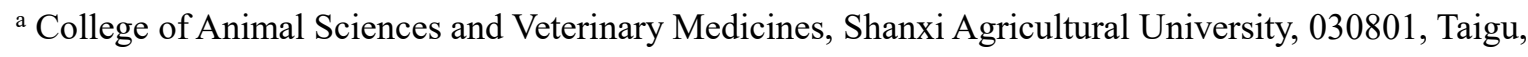 \\ Shanxi Province, P. R. China
}

${ }^{\mathrm{b}}$ Feed and Veterinary Medicine Research Institute, Shanxi Academy of Agriculture Sciences, 030036, Taiyuan, Shanxi Province, P. R. China

${ }^{\mathrm{c}}$ Department of Clinical Neurosciences, UCL Institute of Neurology, Rowland Hill Street, London NW3 2PF, UK

* Corresponding author: Shi-yu Yang, e-mail address: shiyu.yang@ucl.ac.uk, Qiang Liu, e-mail address:

\section{liuqiangabc@163.com}

\# These authors have contributed equally to this work.

\section{Highlights:}

- Neural crest stem cells are attractive candidates for medical application

- A separation strategy was developed to isolate the cells in adipose tissues easily

- The cell are able to self-renew and form neurosphere ex vivo

- Adipose tissues offer an ideal resource to get Neural crest stem cells efficiently

\begin{abstract}
Neural crest stem cells (NCSCs) often referred to as the fourth germ layer, comprise a migratory, stem and progenitor cell population and are synonymous with vertebrate evolution and development. The cells follow specific paths to migrate to different locations of the body where they generate a diverse array of cell types and tissues. There are NCSCs which are maintained
\end{abstract}


in an undifferentiated state throughout the life in the animal tissues. Based on some cells migratory property, we successfully developed a separation strategy to isolate and identify a population of adipose-derived stem cells with neural crest stem cell features in adult bovine adipose tissues within minimally-invasive surgical procedures. The cells have a high degree of multi-potency and self-renewal capabilities, can be cultured and maintained in feeder-free adhesion conditions as monolayer cells, and also be able to grow in the suspension condition in the form of neurosphere. For the purpose of simple description, we name this type cell as bovine adipose-derived neural crest stem cell (baNCSC). Taken together our study describes a readily accessible source of multipotent baNCSC for autologous tissue engineer and cell-based therapeutic researches.

KEYWORDS neural crest-derived stem cells; adipose; bovine; multipotent; differentiation

\section{INTRODUCTION}

Neural crest cells (NCCs) are a transient population of highly migratory multipotential precursors raised between the epidermis and the neural tube during the early stage of vertebrate development [1]. The latest finding suggests that NCCs evolve from lateral plate ectoderm preceded the advent of vertebrates [2]. Following individualization in the dorsal-the most part of the closing neural tube, the NCCs undergo epithelial-to-mesenchymal transition (EMT) $[3,4]$, delaminate and migrate along several stereotyped routes within embryonic tissues and finally settle and differentiate in various elected location [5]. NCCs have the capacity of self-renew [6] with remarkable invasion properties and lineage repertoire which allow them to be able to populate virtually all the tissues and generate a wide diversity of neural and non-neural cell types $[7,8]$. Phenotypic differentiation of NCCs is strongly modulated by their spatial identity 
and the onset of migration for responding to the environmental cues [9]. Therefore neural crest has been regarded as the original source of all adult stem cells [10]. Stemple et al. [11] isolated and enriched NCCs from explanted rat embryologic truck neural tubes and demonstrated the multipotency and self-renewable properties of NCCs and therefore named them as Neural Crest-Derived Stem Cells (NCSCs).

NCSCs have been identified from various adult vertebrate tissues [12], such as skin [13], cornea [14], heart [15], carotid body [16], Whisker Pad [5], craniofacial [17] and adipose tissues [18]. Thanks to their multipotency, high proliferation rate and the lack of major ethical limitations, NCSCs have been made attractive candidates for medical application, such as setting up a research cellular modelling of adipogenic biology, forming a potential therapeutic plan of neural degenerative disease and tissue regeneration. Notably, according to the revised ISSCR guidelines for stem cell research and clinical translation, large animal models should be used since they better emulate human anatomy and/or pathology [19]. Comparing with rodent, cattle's genome organization is more similar to human because of most cattle chromosomes primarily corresponding to part of one human chromosome [20], it means that cattle can replace rodent as animal models for studying human diseases.

\section{MATERIALS AND METHODS}

\subsection{Used medium}

Isolation and growth medium: DMEM (High Glucose, Glutamax, Gibco) supplemented with foetal bovine serum (10\%, Gibco), Sodium Pyruvate (1mM, Sigma), Uridine (50ug/mL, Sigma), Pen strep (50 units/mL, Gibco), Fungizone (Amphotericin B, 1.25 ug/mL, Gibco).

Cryopreservation medium: Isolation and growth medium plus 10\% DMSO (Sigma). 
Neurosphere formation medium: DMED/F12 (1:1, Glutamax, Gibco) supplemented with B27 supplement (1x, Gibco), Recombinant human FGF basic (20ng/mL, R\&D), Recombinant human EGF (10ng/mL, Bio-Rad), Recombinant human leukemia inhibitory factor $(10 \mathrm{ng} / \mathrm{mL}$, Sigma), Pen strep (50 units/mL), Fungizone (Amphotericin B, 1.25 ug/mL, Gibco).

Adipogenic differentiation medium: $\alpha$-MEM (Gibco) supplemented with adipogenic supplement (1\%, R\&D), foetal bovine serum (10\%, Gibco), Pen strep (50 units/mL, Gibco), Fungizone (Amphotericin B, $1.25 \mathrm{ug} / \mathrm{mL}$, Gibco).

Osteogentic differentiation medium: $\alpha$-MEM (Gibco) supplemented with osteogenic supplement (5\%, R\&D), foetal bovine serum (10\%, Gibco), Pen strep (50 units/mL, Gibco), Fungizone (Amphotericin B, $1.25 \mathrm{ug} / \mathrm{mL}$, Gibco).

Chondrogenic differentiation medium: DMED/F12 (1:1, Glutamax, Gibco) supplemented with chondrogenic supplement (1\%, R\&D) and ITS supplement (1\%, R\&D).

\section{2 baNCSCs isolation, culture and passage procedure}

The subcutaneous adipose tissues were obtained using minimally-invasive ablation by biopsy from healthy Chinese jinnan yellow cattle in concordance with ethics board approvals for the research by Laboratory Animal Ethics Committee Shanxi Agricultural University (Number 20181210-02). The normal fat sample was washed with PBS (Hyclone), dissected to small pieces (less than $0.2 \mathrm{~cm}$ diameter) in the growth medium, subsequently explanted in well precoated with collagens; a sterilized coverslip was used to cover the tissue to prevent it from floating. The medium was changed every 4 days until migrated cells reach to a certain density. Cells were dissociated from well with accutase (Sigma) and seeded in normal culture dishes with the same medium at $37^{\circ} \mathrm{C}$ with $5 \% \mathrm{CO}_{2} / 95 \%$ air and passaged with a ratio of $1: 3$ when 
cell density reach to $70-80 \%$ confluence.

\section{3 baNCSCs differentiation protocol}

Adipogenic differentiation protocol: A sterile coverslip was inserted into each well of 24-well plate. $0.5 \mathrm{~mL}$ of baNCSCs $\left(7.4 \times 10^{4}\right.$ cells $\left./ \mathrm{mL}\right)$ suspension was dispensed into each well. After cells are $100 \%$ confluent, the medium was replaced with adipogenic differentiation medium. Cells were incubated at $37^{\circ} \mathrm{C}$ with $5 \% \mathrm{CO} 2 / 95 \%$ air. The medium was replaced with fresh made adipogenic differentiation medium every 4 days. Following 7-14 days differentiation, the cells were fixed with 4\% paraformaldehyde in PBS and saved for immunostaining or red oil staining. Osteogenic differentiation protocol: A sterile coverslip was inserted into each well of 24-well plate and coated with $0.5 \mathrm{~mL}$ fibronectin solution $(1 \mathrm{ug} / \mathrm{mL})$ per well. $0.5 \mathrm{ml}$ of baNCSCs $\left(7.4 \times 10^{4}\right.$ cells $\left./ \mathrm{mL}\right)$ cell suspension was dispensed into each well and incubated at $37^{\circ} \mathrm{C}$ with $5 \%$ $\mathrm{CO}_{2} / 95 \%$ air. At $50-70 \%$ confluent, the $\alpha$-MEM medium was replaced with osteogenic differentiation medium. Cells were cultured with $5 \% \mathrm{CO}_{2} / 95 \%$ air. The medium was replaced with fresh osteogenic differentiation medium $(0.5 \mathrm{~mL})$ every 4 days. After $14-21$ days, cells were fixed with $4 \%$ paraformaldehyde in PBS and saved for immunostaining or Alizarin red S (ARS) staining.

Chondrogenic differentiation protocol: Chondrogenic differentiation medium was made freshly. $2.5 \times 10^{5}$ cells in the growth medium were transferred to a $15 \mathrm{~mL}$ conical tube. The cells were pelleted by centrifuging at $200 \times g$ for 5 minutes at room temperature. The medium was removed; cells were re-suspended with $0.5 \mathrm{~mL}$ of chondrogenic differentiation medium and pelleted by centrifuging again. The cap of the tube was loosed to allow gas exchange. The cells in the pellet were cultured about 14-21 days. The harvested chondrocyte pellet was fixed, 
cryostat sectioned $(10 \mu \mathrm{m})$ and subjected to immunostaining.

Neurosphere formation and staining: When reached to $70-80 \%$ confluence, baNCSCs were collected and pelleted by centrifuging at $1000 \mathrm{rpm}$ for 10 minutes. The cell pellet was suspended with a small volume of neurosphere formation medium, transferred to uncoated 6 well plate and incubated. The medium was changed every 5 days until a sufficient number of neurospheres formed. The neurospheres were collected for the histological and immunocytochemistry examination.

\subsection{The real-time reverses quantitative (qRT-PCR)}

Total RNA was extracted from cell pellet using RNeasy mini kit with on-column DNase digestion according to the manufacture's guidelines(Qiagen). cDNA were generated from 1.0 $\mu \mathrm{g}$ of RNA with reverse transcription kit (Qiagen). All qPCR reactions were performed as triplicate using power SYBR Green PCR master mix (Life technologies) and assayed with Step One real time PCR system (Applied Bio-system). Primers information is given in Table 1

\subsection{Immunocytochemistry}

Immunocytochemical analysis was performed as described[21]. The details of primary and secondary antibodies and their dilution can be found in the table 2 .

\subsection{Statistical analysis}

Statistical analyses between groups were carried out by GraphPad Prism 6 using a Student's ttest. $P<0.05$ was considered to indicate a statistically significant difference.

\section{RESULT}

\subsection{Isolation of adipose-derived stem cells from bovine adipose tissue}


The bovine adipose tissue was extracted using minimally-invasive access from the subcutaneous fat and explanted with a coverslip covered (Figure 1A). After 5 days the stem cells could be seen migrating from fat tissues and exhibited a typical spindle shape (Figure 1B and C). After 8 days of explanting, cells were ready for harvest (Figure 1D and E). Using the method, the stem cells had been successively isolated, then passaged and cryopreserved.

\subsection{Adipose-derived stem cells express neural crest cell and pluripotent genes}

The result demonstrates that P75NTR and SOX10 are expressed significantly higher compared to the MDBK under feeder-free conditions. (bovine kidney cells, from China Infrastructure of Cell Line Resource) $(P<0.05)$ (Figure 2A and B). The positive staining with P75NTR and SOX10 markers by immunocytochemistry confirm the increase of the two genes expression (Figure 2C and D). Compared with bovine kidney cells, the levels of SOX2 and KFL4 expression are significantly higher in adipose-derived stem cells $(P<0.05)$ (Figure 2E, and 2F). All these data together suggest most of cells isolated with our method form bovine adipose tissues retain neural crest cells features; especially hold the important features of migration and multipotency. We name this type cell as bovine adipose-derived neural crest stem cell (baNCSC).

\section{3 baNCSCs are capable of forming neurospheres and have multipotent features}

Following 24 and 48 hours incubation with sphere-forming medium, the morphology of most cells appeared for the preparation of sphere formation (Figure 3A and B). After 72 hours incubation, the properly neurospheres were formed (Figure 3C). Maintaining with the medium for 144 hours, baNCSCs showed more neurospheres morphology and many various sized white 
suspension neurospheres could be found in the 6-well plate (Figure 3D). The immunocytochemistry staining confirms that the neurospheres are expressing OCT4, SOX2 and SOX10 markers (Figure 3E, F and G) indicating the stem cells keeping multipotent features in the neurospheres.

\subsection{Differentiation capacities of baNCSCs}

baNCSCs were exposed to adipogenic differentiation medium for 2 weeks, most cells were induced to adipogenic cells with fatty vacuous showed by Oil red staining (Figure 4A) and expressed specific adipogenic protein: fatty acid binding protein 4 (FABP-4) (Figure 4B). After cultured with osteogenic differentiation medium for 3 weeks, baNCSCs were observed to express osteocalcin (Figure 4D), a protein secreted solely by osteoblasts, and to have a high calcium deposits and mineralization showed by Alizarin red staining (Figure 4C). After cultured in chondrogenic differentiation medium in the form of cellular nodules for 2 weeks, the cellular nodule formed a proper cartilage-like tissue structures (Figure 4E); and baNCSC cells produced a high level of cartilage-related extracellular matrix molecule: aggrecan (Figure 4F). All these data shown baNCSCs are able to differentiate to multiple cell lineages in vitro when cultivated in the appropriate differentiation media.

\section{DISCUSSION}

\subsection{Isolation of adipose-derived stem cells from bovine adipose tissue}

There are many different types of adult stem cells existing in adipose tissue [22,23]. Several investigators have successfully identified stem cell niche in adipose tissues and found adipose stem cells reside in a vascular niche embedded with the wall of blood vessels that supply fat tissues $[24,25]$. Because of low oxygen concentration and limited proliferative activity in stem 
cell niches, stem cell energy supply is more dependent on glycolysis rather than on OXPHOS [26]. Unlike the procedures traditionally used in isolation of stem cells from adipose tissue which involved with a big volume of fat tissues and enzymatic digestion, we provide energyrich medium to enhance stem cells migrating from adipose tissue. Utilizing some cells migratory property, we have successively isolated stem cells from 20 individual bovine adipose samples with nearly $100 \%$ success rate and observed that they are able to self-renew ex vivo under feeder-free conditions. Because the tissue volume used in the procedure is small, only migrated cells are isolated, the stem cells have advantages in terms of high purity and less contamination compared with the traditional separation methods. Previous research suggest that the resistance to stable proliferation appears to be a general challenge in the stem cells[9,27], thus the stability of the bovine adipose-derived stem cells may require further characterized.

\subsection{Adipose-derived stem cells express pluripotent and neural crest cell genes}

As the adult somatic cells deriving from the intermediate mesoderm, MDBK $d$ reach a state of terminal differentiation and the plasticity of the cells generally are regarded as low to nonexistent, or the potential can be reprogrammed under specific conditions [28]; Therefor the MDBK was used as a control group. High expression of P75NTR and SOX10 genes is one of the typical neural crest stem cells characters, which have been used to isolate NCSCs through flow cytometry analysis in the cell outgrowth from neural tube [11]. The genes and protein high expression suggested that there are stem cells with neural crest stem cell features in adult bovine adipose tissues. OCT4, SOX2, and KLF4 have been regarded as the typical endogenous pluripotent genes in human iPS stem cells[29]. Previous study notice NCSCs may have evolved as a consequence of a subset of blastula cells retaining activity of core pluripotency genes [30]. 
We demonstrate that SOX2 and KLF4 are expressed at obviously higher levels in baNCSCs compared with bovine kidney cells. KLF4 is a transcriptional regulator of genes critical for epithelial-mesenchymal transition [31], which is pre-step of neural crest cells migrating to various elected locations during embryo development [5]. Therefore it will not be surprised to expect that the expression level of KLF4 would be pronounced higher in baNCSCs than adult somatic cells. ESCs with reduce OCT4 expression resulting from heterozygosity exhibit a stabilized pluripotent state through enhancing self-renewal efficiency and delaying differentiation kinetics [32]. The similar strategies may be used in NCSCs systems to facilitate rapid responses to changing physiological environments. Therefore it would make sense for these stem cells decrease OCT4 expression.

\section{3 baNCSCs are capable of forming neurospheres and have multipotent features}

It has been established that the neural crest-derived tissues are capable of forming neurospheres[13,15,33]. The sphere-forming capability has been used to test ontogeny and multipotency of NCSCs derived from bone marrow, dorsal root ganglia and whisker pad [5]. In order for NCCs to migrate to verity locations of the embryo during development, NCCs are required to transit its epithelial morphology to mesenchymal morphology (epithelialmesenchymal transition, EMT) [1]. The reverse process of EMT is mesenchymal-epithelial transition (MET), MET is an essential step for fibroblast to be induced to pluripotency stem cells (iPSCs) [34]. We transferred monolayer baNCSCs cells to neurosphere which is the MET process, the pluripotency markers including OCT4, SOX2, and SOX10 still exist.

\subsection{Differentiation capacities of baNCSCs}

Previous researches investigated the relationship between NCSCs and mesenchymal stem cell 
(MSC) and find that MSCs in BM are partially originated from NCSCs [35,36]. MSC is a type of stem cells that undergo sustained in vitro growth and is able to differentiate to multiple mesenchymal lineages[37,38]. In addition, our in vitro data prove NCSCs coming from adipose tissue have high phenotypic plasticity. This is also constant with the previous observation that a large proportion of trunk NCCs are able to yield a large variety of fully differentiated ectomesenchyme-derived cell types (bone, cartilage, smooth muscle, and adipose cells), together with neural (neurons, glia) and pigment cells [8,39]. Yang et al. [18] have developed dopaminergic neuronal lines from NCSCs (derived from human adipose tissues) to invest the disease-modifying therapies in Parkinson's disease. The NCSC approach has advantages in terms of ease of access to neural crest cells and retention of the intact host genome. Thus, NCSCs are anticipated to open enormous opportunities in the biomedical sciences in terms of cell therapies for degeneration diseases and cellular modeling of animal diseases.

\section{ACKNOWLEDGMENTS}

This work was supported by the eighth group of overseas high-level personnel of Shanxi province introduction project.

\section{REFERENCES}

[1] A. Achilleos, P.A. Trainor, Neural crest stem cells: discovery, properties and potential for therapy, Cell Res 22 (2012) 288-304. http://doi.org/10.1038/cr.2012.11.

[2] R. Horie, A. Hazbun, K. Chen, et al., Shared evolutionary origin of vertebrate neural crest and cranial placodes, Nature 560 (2018) 228-232. http://doi.org/10.1038/s41586-018-0385-7.

[3] E. Dupin, S. Creuzet, N.M. Le Douarin, The contribution of the neural crest to the vertebrate body, Adv Exp Med Biol 589 (2006) 96-119. http://doi.org/10.1007/978-0-387-46954-6 6.

[4] A.V. Morales, J.A. Barbas, M.A. Nieto, How to become neural crest: from segregation to delamination, Seminars in cell \& developmental biology 16 (2005) 655-662. http://doi.org/10.1016/i.semcdb.2005.06.003.

[5] N. Nagoshi, S. Shibata, Y. Kubota, et al., Ontogeny and multipotency of neural crest-derived stem cells in mouse bone marrow, dorsal root ganglia, and whisker pad, Cell Stem Cell 2 (2008) 392-403. http://doi.org/10.1016/i.stem.2008.03.005.

[6] K. Janebodin, O.V. Horst, N. leronimakis, et al., Isolation and characterization of neural crest-derived stem cells 
from dental pulp of neonatal mice, PLoS One 6 (2011) e27526. http://doi.org/10.1371/journal.pone.0027526.

[7] E. Dupin, J.M. Coelho-Aguiar, Isolation and differentiation properties of neural crest stem cells, Cytometry. Part A : the journal of the International Society for Analytical Cytology 83 (2013) 38-47. http://doi.org/10.1002/cyto.a.22098.

[8] E. Dupin, L. Sommer, Neural crest progenitors and stem cells: from early development to adulthood, Dev Biol 366 (2012) 83-95. http://doi.org/10.1016/i.ydbio.2012.02.035.

[9] S. Münst, P. Koch, J. Kesavan, et al., In vitro segregation and isolation of human pluripotent stem cell-derived neural crest cells, 2017.

[10] C. Pierret, K. Spears, J.A. Maruniak, et al., Neural crest as the source of adult stem cells, Stem cells and development 15 (2006) 286-291. http://doi.org/10.1089/scd.2006.15.286.

[11] D.L. Stemple, D.J. Anderson, Isolation of a stem cell for neurons and glia from the mammalian neural crest, Cell 71 (1992) 973-985.

[12] E. Dupin, G.W. Calloni, J.M. Coelho-Aguiar, et al., The issue of the multipotency of the neural crest cells, Dev Biol 444 Suppl 1 (2018) S47-S59. http://doi.org/10.1016/i.ydbio.2018.03.024.

[13] K.J. Fernandes, I.A. McKenzie, P. Mill, et al., A dermal niche for multipotent adult skin-derived precursor cells, Nat Cell Biol 6 (2004) 1082-1093. http://doi.org/10.1038/ncb1181.

[14] S. Yoshida, S. Shimmura, N. Nagoshi, et al., Isolation of multipotent neural crest-derived stem cells from the adult mouse cornea, Stem Cells 24 (2006) 2714-2722. http://doi.org/10.1634/stemcells.2006-0156.

[15] Y. Tomita, K. Matsumura, Y. Wakamatsu, et al., Cardiac neural crest cells contribute to the dormant multipotent stem cell in the mammalian heart, J Cell Biol 170 (2005) 1135-1146. http://doi.org/10.1083/icb.200504061.

[16] R. Pardal, P. Ortega-Saenz, R. Duran, et al., Glia-like stem cells sustain physiologic neurogenesis in the adult mammalian carotid body, Cell 131 (2007) 364-377. http://doi.org/10.1016/i.cell.2007.07.043.

[17] W.D. Grimm, A. Dannan, B. Giesenhagen, et al., Translational Research: Palatal-derived Ecto-mesenchymal Stem Cells from Human Palate: A New Hope for Alveolar Bone and Cranio-Facial Bone Reconstruction, International journal of stem cells 7 (2014) 23-29. http://doi.org/10.15283/ijsc.2014.7.1.23.

[18] S.Y. Yang, M. Beavan, K.Y. Chau, et al., A Human Neural Crest Stem Cell-Derived Dopaminergic Neuronal Model Recapitulates Biochemical Abnormalities in GBA1 Mutation Carriers, Stem Cell Reports 8 (2017) 728-742. http://doi.org/10.1016/i.stemcr.2017.01.011.

[19] G.Q. Daley, I. Hyun, J.F. Apperley, et al., Setting Global Standards for Stem Cell Research and Clinical Translation: The 2016 ISSCR Guidelines, Stem Cell Reports 6 (2016) 787-797. http://doi.org/10.1016/i.stemcr.2016.05.001.

[20] C.G. Elsik, R.L. Tellam, K.C. Worley, et al., The genome sequence of taurine cattle: a window to ruminant biology and evolution, Science 324 (2009) 522-528. http://doi.org/10.1126/science.1169588.

[21] L. Hyslop, M. Stojkovic, L. Armstrong, et al., Downregulation of NANOG induces differentiation of human embryonic stem cells to extraembryonic lineages, Stem Cells 23 (2005) 1035-1043. http://doi.org/10.1634/stemcells.2005-0080.

[22] J.M. Gimble, A.J. Katz, B.A. Bunnell, Adipose-derived stem cells for regenerative medicine, Circ Res 100 (2007) 1249-1260. http://doi.org/10.1161/01.RES.0000265074.83288.09.

[23] P.A. Zuk, M. Zhu, P. Ashjian, et al., Human adipose tissue is a source of multipotent stem cells, Molecular biology of the cell 13 (2002) 4279-4295. http://doi.org/10.1091/mbc.e02-02-0105.

[24] M.S. Rodeheffer, K. Birsoy, J.M. Friedman, Identification of white adipocyte progenitor cells in vivo, Cell 135 (2008) 240-249. http://doi.org/10.1016/i.cell.2008.09.036.

[25] T. Shan, W. Liu, S. Kuang, Fatty acid binding protein 4 expression marks a population of adipocyte progenitors 
in white and brown adipose tissues, FASEB J 27 (2013) 277-287. http://doi.org/10.1096/fj.12-211516.

[26] Z. Ivanovic, Hypoxia or in situ normoxia: The stem cell paradigm, J Cell Physiol 219 (2009) 271-275. http://doi.org/10.1002/icp.21690.

[27] G. Lee, H. Kim, Y. Elkabetz, et al., Isolation and directed differentiation of neural crest stem cells derived from human embryonic stem cells, Nat Biotechnol 25 (2007) 1468-1475. http://doi.org/10.1038/nbt1365.

[28] C. Shi, T. Cheng, Y. Su, Exploring the developmental potential of adult mammalian somatic cells, Chinese Science Bulletin 46 (2001) 4-5. http://doi.org/10.1007/bf03183198.

[29] H. Jiang, Y. Ren, E.Y. Yuen, et al., Parkin controls dopamine utilization in human midbrain dopaminergic neurons derived from induced pluripotent stem cells, Nat Commun 3 (2012) 668. http://doi.org/10.1038/ncomms1669.

[30] E. Buitrago-Delgado, K. Nordin, A. Rao, et al., Shared regulatory programs suggest retention of blastula-stage potential in neural crest cells, Science 348 (2015) 1332-1335. http://doi.org/10.1126/science.aaa3655.

[31] N. Tiwari, N. Meyer-Schaller, P. Arnold, et al., Klf4 is a transcriptional regulator of genes critical for EMT, including Jnk1 (Mapk8), PLoS One 8 (2013) e57329. http://doi.org/10.1371/journal.pone.0057329.

[32] V. Karwacki-Neisius, J. Goke, R. Osorno, et al., Reduced Oct4 expression directs a robust pluripotent state with distinct signaling activity and increased enhancer occupancy by Oct4 and Nanog, Cell Stem Cell 12 (2013) 531-545. http://doi.org/10.1016/i.stem.2013.04.023.

[33] L. Kerosuo, S. Nie, R. Bajpai, et al., Crestospheres: Long-Term Maintenance of Multipotent, Premigratory Neural Crest Stem Cells, Stem Cell Reports 5 (2015) 499-507. http://doi.org/10.1016/i.stemcr.2015.08.017.

[34] R. Li, J. Liang, S. Ni, et al., A mesenchymal-to-epithelial transition initiates and is required for the nuclear reprogramming of mouse fibroblasts, Cell Stem Cell 7 (2010) 51-63. http://doi.org/10.1016/i.stem.2010.04.014. [35] M. Fukuta, Y. Nakai, K. Kirino, et al., Derivation of mesenchymal stromal cells from pluripotent stem cells through a neural crest lineage using small molecule compounds with defined media, PLoS One 9 (2014) e112291. http://doi.org/10.1371/journal.pone.0112291.

[36] S. Morikawa, Y. Mabuchi, K. Niibe, et al., Development of mesenchymal stem cells partially originate from the neural crest, Biochem Biophys Res Commun $379 \quad$ (2009) 1114-1119. http://doi.org/10.1016/i.bbrc.2009.01.031.

[37] J.P. Darwin, Marrow stromal cell as stem cells for nonhematopoietic tissues, Science 276 (1997) 71-74.

[38] M.F. Pittenger, A.M. Mackay, S.C. Beck, et al., Multilineage potential of adult human mesenchymal stem cells, Science 284 (1999) 143-147. http://doi.org/10.1126/science.284.5411.143.

[39] S. Thomas, M. Thomas, P. Wincker, et al., Human neural crest cells display molecular and phenotypic hallmarks of stem cells, Hum Mol Genet 17 (2008) 3411-3425. http://doi.org/10.1093/hmg/ddn235.

\section{FIGURE/TABLE LEGENDS}

Figure 1. Isolation of stem cells from bovine adipose tissue. (A) The subcutaneous adipose tissue was explanted and covered by a coverslip in the medium. (B, C) The adipose-derived stem cell could be seen migrating from fat tissues in 5 days (original magnification $\times 5$ and $\times 10$ ). $(\mathrm{D}, \mathrm{E})$ The more adipose-derived stem cell migrated in 8 days (original magnification $\times 5$ and $\times 10$ ), then we detached cells using enzymatic methods, passaged and cryopreserved. Images were acquired using a stereomicroscope (Leica DMIL LED). 
Figure 2. Characterization of in vitro expanded Adipose-derived stem cells. (A, B) Quantitative assessment of NCSCs hallmarks genes including P75NTR and SOX10 mRNA expression comparing with MDBK. $* * P<0.05$ versus MDBK. (C, D) The anti-p75NTR antibody (green) and anti-SOX10 antibody (red) were employed for immunocytochemistry. Nuclear counterstaining was performed using DAPI (blue). All images were captured using the Pathway Bioimager System (Lecia, DM2500). (E, F, G) Quantitative assessment of pluripotent genes including KLF4, SOX2, and OCT4 mRNA expression. ${ }^{* *} P<0.05$ versus MDBK.

Figure 3. The process of baNCSCs forming neurospheres and expression of pluripotent genes. (A, B, C, D) baNCSCs formed neurospheres in $24 \mathrm{~h}, 48 \mathrm{~h}, 72 \mathrm{~h}$, and $144 \mathrm{~h}$ under serum-free culture condition. (E), (F), (G) Immunohistochemical evaluation of neurospheres on OCT4 (green), SOX2 (green), and SOX10 (red) expression at protein level in vivo, nuclear counterstaining was performed using DAPI (blue).

Figure 4. Differentiation of baNCSCs into adipogenic, osteogenic and chondrogenic cell types. (A) Adipogenic differentiation was indicated by the presence of lipid droplets stained with Oil Red O. (B) Adipogenic expressed FABP4 demonstrated by immunostaining. (C) Osteocyte differentiation was assessed by staining calcium-rich areas with Alizarin red and the shape changed from fusiform to triangular, (D) anti-hOsteocalcin antibody was used for evidence the osteogenic differentiation. (E) Chondrogenic pellet formed in the tube, (F) anti-Aggrecan antibody demonstrated the chondrogenic differentiation. 


\section{Table 1}

The primers used for real time QRT-PCR reactions.

\begin{tabular}{ccc}
\hline Genes & Primer sequences & Product length \\
\hline P75NTR & f. 5'CCTGTCTATTGCTCCATCCTG 3' & $101 \mathrm{bp}$ \\
SOX10 & r. 5' TTGGCTCCTTGCTTGTTCTG 3' & \\
& f. 5' CCCCAAAGACAGAGCTGCAG 3' & $104 \mathrm{bp}$ \\
OCT4 & r. 5' GATGTCCACGTTGCCGAAGT 3' & \\
& f. 5' CCTGTCTATTGCTCCATCCTG 3' & $87 \mathrm{bp}$ \\
SOX2 & r. 5' GTCGTTTGGCTGAACACCTT 3' & \\
& f. 5' CCCTGCAGTACAACTCTATGACC 3' & $175 \mathrm{bp}$ \\
KLF4 & r. 5' CTGGAGTGGGAAGAAGAGGTAA 3' & \\
& f. 5' GGAGACGGAGGAGTTCAATGAT 3' & $118 \mathrm{bp}$ \\
GADPH & r. 5' GGACGAGGATGAGGCTGATG 3' & \\
& f. 5'TGAACCACGAGAAGTATAACAACAC 3' & $125 \mathrm{bp}$ \\
\hline
\end{tabular}

Table 2

The antibodies used for immunocytochemistry.

\begin{tabular}{|c|c|c|}
\hline Proteins & primary antibody & secondary antibodies \\
\hline P75NTR & rabbit anti-P75NTR (Bioss) (1:100) & $\begin{array}{l}\text { goat anti-rabbit Texas lgG/FITC (Bioss) } \\
(1: 200)\end{array}$ \\
\hline SOX10 & $\begin{array}{l}\text { mouse anti-SOX10 (Santa Cruz) } \\
(1: 200)\end{array}$ & $\begin{array}{l}\text { donkey anti-mouse } \operatorname{lgG} \text { NorthernLights }{ }^{\mathrm{TM}} \\
\text { NL557 (R\&D) (1:200) }\end{array}$ \\
\hline OCT4 & rabbit anti-OCT4 (Abcam) $(1: 250)$ & $\begin{array}{l}\text { goat anti-rabbit lgG H\&L (Alexa Fluor } \mathbb{B} 488) \\
(\text { Abcam) }(1: 200)\end{array}$ \\
\hline $\mathrm{SOX} 2$ & rabbit anti-SOX2 (Abcam) $(1: 200)$ & $\begin{array}{l}\text { goat anti-rabbit } \operatorname{lgG} \text { H\&L (Alexa Fluor } B 488) \\
\text { (Abcam) (1:200) }\end{array}$ \\
\hline FABP4 & goat anti-mFABP4 $(\mathrm{R} \& \mathrm{D})(10 \mu \mathrm{g} / \mathrm{mL})$ & $\begin{array}{l}\text { donkey anti-goat } \operatorname{lgG} \text { NorthernLights }{ }^{\mathrm{TM}} \\
\text { NL557 (R\&D) (1:200) }\end{array}$ \\
\hline Aggrecan & $\begin{array}{l}\text { goat anti-hAggrecan (R\&D) } \\
(10 \mu \mathrm{g} / \mathrm{mL})\end{array}$ & $\begin{array}{l}\text { donkey anti-goat lgG } \text { NorthernLights }^{\mathrm{TM}} \\
\text { NL557 (R\&D) (1:200) }\end{array}$ \\
\hline Osteocalcin & $\begin{array}{l}\text { mouse anti-hOsteocalcin (R\&D) } \\
(10 \mu \mathrm{g} / \mathrm{mL})\end{array}$ & $\begin{array}{l}\text { donkey anti-mouse } \operatorname{lgG} \text { NorthernLights }{ }^{\mathrm{TM}} \\
\text { NL557 (R\&D) }(1: 200)\end{array}$ \\
\hline
\end{tabular}


Figure 1
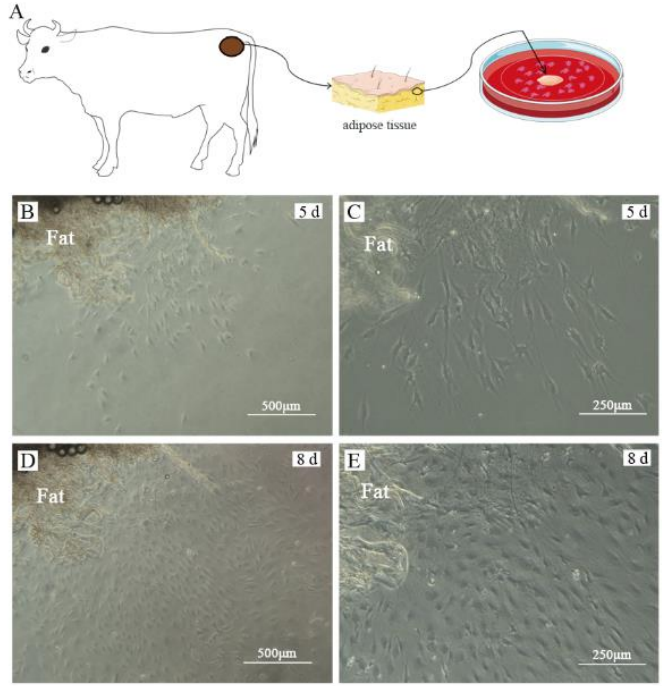

Figure 2
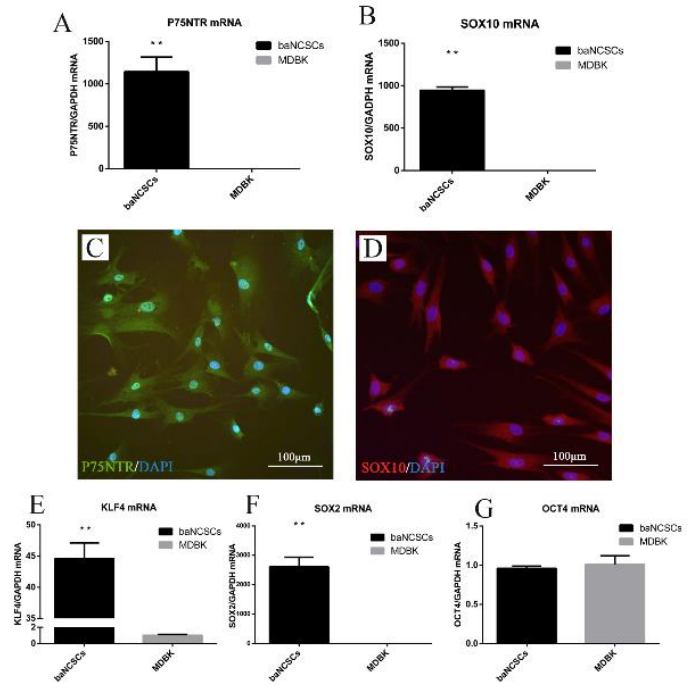

Figure 3

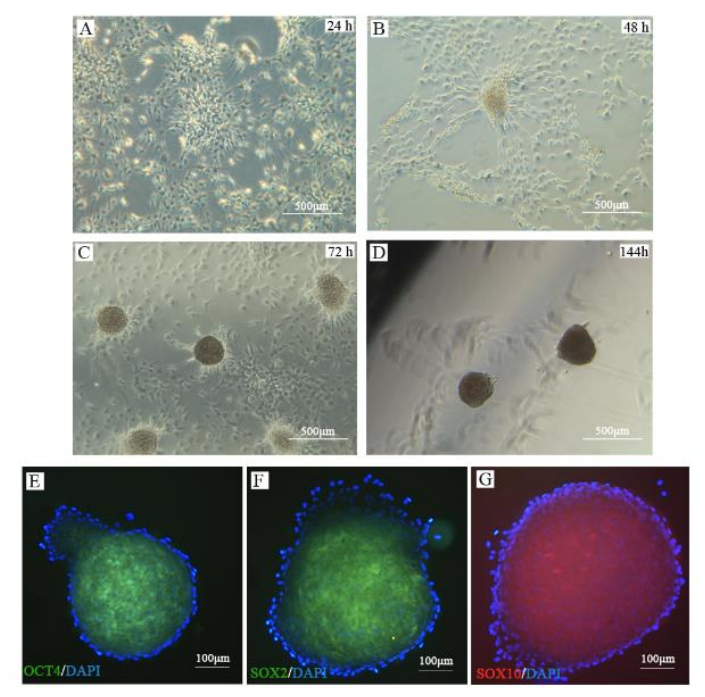


Figure 4

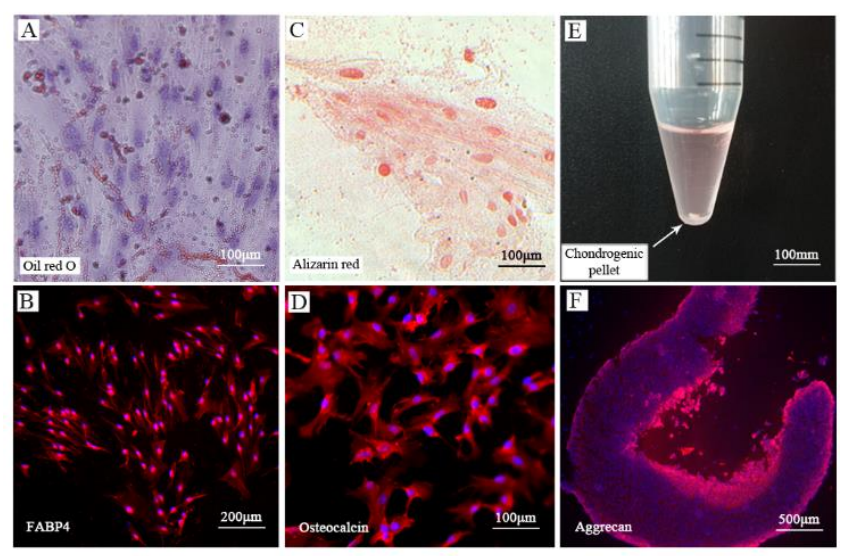

